\title{
Research regarding electro-oculogram based Human Computer Interface (HCI)
}

\author{
Nagy Robert-Béla \\ University of Oradea \\ Oradea, Romania \\ nagyrobertbela@gmail.com
}

\author{
Tiberiu Vesselenyi \\ University of Oradea \\ Oradea, Romania \\ tvesselenyi@yahoo.co.uk
}

\begin{abstract}
In this article an electro-oculogram (EOG) based Human Computer Interface (HCI) will be presented, in order to control the mouse cursor on the screen of a computer or laptop. Electromyography (EMG) is the domain that employs the activation and deactivation (onset and cessation) of the muscles. EOG is the sub-domain of EMG field that focuses on the human eye's movements. The EOG bio-signals can be recorded using $\mathrm{Ag} / \mathrm{AgCl}$ electrodes coupled on the user's skin and fed into a data acquisition device - an analog-to-digital converter (ADC) in order to be transmitted, filtered and processed on a computer or laptop. We acquired the EOG bio-signals with a 24-bit, 4 channel, 51200 samples/s per channel ADC, made by the National Instruments (N.I.), model NI-9234 industrial ADC, using only 3 recording channels and electrodes. After processing, the program running on the computer or laptop can be used to realize commands or control different applications according to the recorded bio-signals. In our case, this was done, using Artificial Neural Network (ANN) toolbox of MATLAB ${ }^{\circledR}$. This HCI can be used by perfectly healthy or even by disabled people. In the case of disabled people, these systems can be used to control any electronic device connected to the computer or control the device itself. Applications of this type of HCIs can be Internet browsing, mail writing, word file editing, etc. This system is meant to offer a new way of computer control - other than the existing standard communication and/or control possibilities (like keyboard and/or mouse).
\end{abstract}

Keywords-Artificial Neural Network, bio-signal acquisition, Electrooculogram, EOG, HCI, mouse control.

\section{INTRODUCTION}

This article presents an electro-oculogram (EOG) based Human Computer Interface (HCI), its realization process, and other details, which, in the end, will be able to control the mouse's cursor on the screen of a computer or laptop, depending on the used hardware.

Electromyography (EMG) is the science that is based on the activation and deactivation (onset and cessation) of the human skeletal muscles. It is used in several applications, from sport and recreation up to electronic device control, and has multiple subdomains. EOG is one of them and it focuses primarily on the human eye's movements. The EOG biosignals are generated by the movement of the human eye in the 4 main directions (left, right, up, down) and are recorded from the skin around the eye of the user. These bio-signals can be recorded using $\mathrm{Ag} / \mathrm{AgCl}$ or any other types of electrodes, like the gold-plated electrodes, reusable electrodes, modified headbands or needle electrodes. In our application we used disposable $\mathrm{Ag} / \mathrm{AgCl}$ electrodes to record these bio-signals.

The $\mathrm{Ag} / \mathrm{AgCl}$ electrodes are coupled on the user's skin, as close as possible to the eyes and the recorded bio-signals can be fed into an analogic filtering system or directly in a digitizer (an analog-to-digital converter - ADC) in order to be transmitted, optionally filtered and further processed on a computer or laptop. After processing, the issued commands of the user can be used in different applications, like Internet browsing, mail writing, word file editing, wheelchairs handling, robot control, recognize reading activity, type in a virtual keyboard, move cursor on the screen, gaming, etc. As it can be seen, this kind of systems can be a new way of communication and/or control for healthy users - being an alternative way to a keyboard or a mouse or it can be used by disabled people. These systems can control any electronic device that is connected to the computer or laptop or even control the device itself.

The other articles from this domain can be considered very wide in terms of fields of use and possibilities of use for the EMG and/or EOG signals. In article [1] different bio-signals (EMG, EOG, Electroencephalogram (EEG), Galvanic skin response and more) are proposed to be used as a possibility of for human recognition. In [2] analyses and a new statistical method to discriminate noise regions from EMG of muscular activities during rhythmic behaviors (using EMG data on mammalian jaw-adductor muscles during mastication) for biomechanical studies are presented. Other application of EMG is in depression observation [3]: the subjects showed reduced responsiveness - as compared to healthy people during reward anticipation and also the punishment anticipation. In their work they recorded facial EMG reactivity, what is very less used in research - according to them.

Other use of EMG signals are mentioned in [4]-[8]: an implementation, which accelerates the computation of different EMG signal's linear envelope was presented in [4]. In [5] they used improved empirical mode decomposition to analyze the bio-signals from patients with amyotrophic lateral sclerosis and comparing it with EMG bio-signals from healthy people. According to [6], the authors could extract the motor 
unit profile, and created an algorithm that detects the turns of the scanning-EMG signal and also can link this, by using point-tracking techniques. In [7] the authors introduced an approach for EMG noise level approximation in electrocardiogram signals, using the stationary wavelet transform. Article [8] presents the proposal of an onset detection method, using offline pathological, weak and noisy EMG signals.

The EMG bio-signals can be used in different applications, as presented above, but it can be also a problem in different domains, like in the EEG domain. Articles [9]-[13] present the other side of EMG and/or EOG bio-signals - they are considered noise that generates artifacts in the EEG bio-signal recording process. All these articles are focused on the removal of these noise sources (EMG and/or EOG) with different processes and results, e.g. in [10] they used wavelet neural network to remove the noise, which combines the generic approximation properties of neural networks with the time/frequency property of the wavelet transform. According to [11], the ocular artifacts (with other words, EOG biosignals) must be removed to obtain a higher accuracy and quality EEG signal. Article [12] is a very extensive and also in-depth review of this topic of removal of EMG and EOG artifacts from EEG bio-signals. They reviewed the current methods for dealing with EMG-EOG generated noise and also and categorized and reviewed more than 250 refereed journal and conference papers from this field.

The domain of EOG bio-signal use is mainly presented in articles [14]-[24]. Articles [14] and [15] presents different use of EOG bio-signals, like evaluating eye movement's characteristics in narcolepsy [14] or drowsiness detection, followed by decisions according to the level of drowsiness [15].

Papers [16]-[24] present shortly and give us insight in the field of using the EOG signals as command and/or control signals. In [16] a combination of eye tracking and facial electromyography (fEMG) during discrete choice experiments is presented; [18] is focused on the interaction with an intelligent environment through two paradigms of HCI. These setups/systems use commercial devices in surface electromyography (sEMG) and EOG application and also in the video-oculography (VOG) bio-signal recording domain. Article [20] aims to solve the problems encountered in current EOG systems when they are used for long periods of time and the users become tired.

In [21] the identification of the characteristic peak amplitudes associated with eye saccades, blinks or winks based on a set of fuzzy logic rules classifier and a deterministic finite automaton is presented. In this application six low-level commands for navigation purposes were employed. Article [22] presents a single channel EOG system employing in-university-made ultrathin and flexible electrodes, which can be practically used to control computer or machine. As found in [23], they have realized an EOG-HCI, which can make the difference between eight eye directions: up, down, right, left, up-right, up-left, down-right and downleft. In [24] another EOG-HCI is presented, recognizing also 8 eye directions, but this setup/system was an in-universitymade wearable HCI device, with a weigh only of $15 \mathrm{~g}$

\section{LAYOUT OF THE SYSTEM}

In our application we recorded the EOG bio-signals with a 24-bit, 4 input channels, 51200 samples/s per channel ADC, made by National Instruments, model code NI-9234; we used only 3 input channels and the afferent electrodes from the 4 input channels that the board can offer. We have done the recording in a non-invasive manner, placing the electrodes on the user's skin near the eyes. After the raw data acquisition took place, we analyzed the EOG bio-signals (filtered of noise

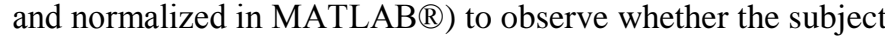
gaze left, right, up or down. The starting point for this work and system is presented and relies on [26].

The next step was to use the in-built Artificial Neural

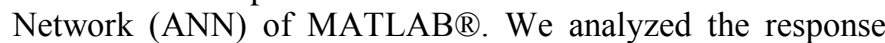
and differentiating capabilities of the trained ANN regarding the recorded EOG signals and also the possibility to create a control input channel for the used laptop to realize the mouse cursor movement actions on the screen with it. Our aim is to make and use this system as a future portable device, which could be also used to help the disabled people (people, who still can control and move their eyes) to control by simple instructions a computer / laptop or any other robotic device connected to it. This system can be used by healthy users too, as was demonstrated in the testing part, offering a new way to control the electronic devices - other than the existing standard communication possibilities (like the keyboard and mouse).

In our design, we used only 3 electrodes to record the EOG bio-signals. Usually 5 electrodes are used in this kind of application, from which one 2 are used to record the vertical eye movements, 2 are used to record the horizontal eye movements and 1 electrode is the reference electrode. In some applications, a 6-th electrode was also used - it was the ground for the system.

EOG signals may contain noise from various sources, such as other electronic or electrical instruments or moving artifacts. While recording, we tried to stop all the unnecessary electronic devices around the user and also the laptop was working only on accumulators.

We used $\mathrm{Ag} / \mathrm{AgCl}$ disposable electrodes in the recording process, as it can be seen in Figure 1. The disposable electrodes are widely used, considering that they can be quickly and easily applied and are also cheap [25]. Disposable electrodes are simply connected by connecting them to a cable that connects it to an ADC. There are many inexpensive types, shaped usually as a disk or rectangle, having glue on its side and contains a central electro-conductive circle made of or coated with $\mathrm{Ag} / \mathrm{AgCl}$. Other disposable electrodes may also contain a semiliquid gel around the central area (this electroconductive gel existed also in our case) in order to create a better conduction. The disadvantage of a large size of the electrode was not a problem in our application. Disposable electrodes may use salt water or saline instead of the gel [25]. In our case the electrodes were clipped to a modified glass, which was used to "carry" the electrodes, as can be seen in 
Figure 2. We could use for this application also the reusable electrodes (which are smaller than the disposable ones), but their advantage to be able to be placed closer to the skin (in areas with a lot of hair) would not help us, and they also cost more than the disposable electrodes.
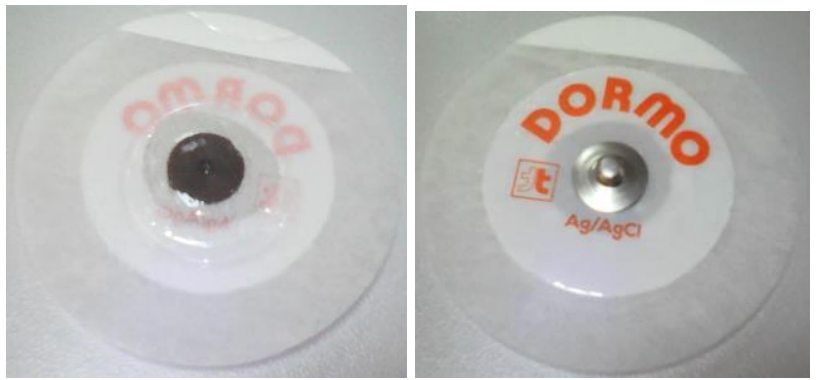

Fig. 1. $\mathrm{Ag} / \mathrm{AgCl}$ electrode used for recording (left - front, right - back)

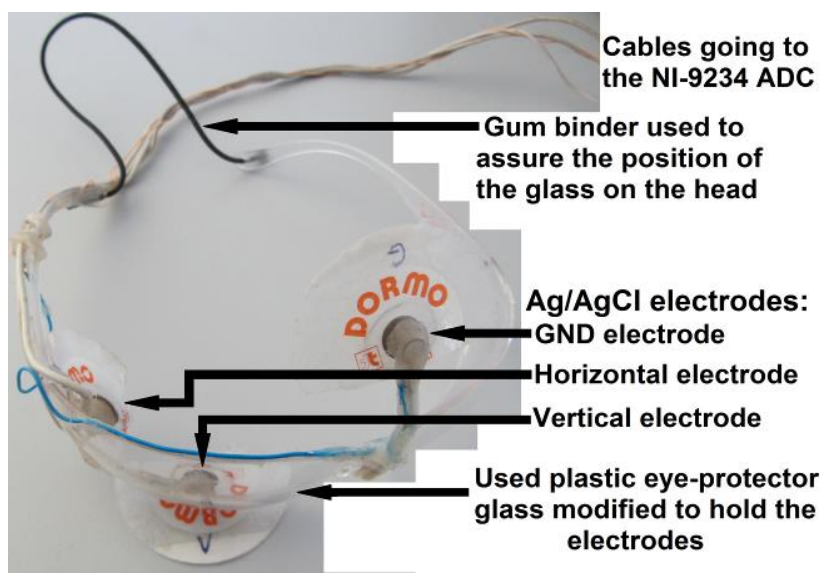

Fig. 2. The modified glass used in the EOG bio-signal's recording process

While recording, the electrodes were numbered, as follows: 0 - Horizontal eye movement input channel, 1 Vertical eye movement input channel, 2 - Ground.

Because we wanted a non-invasive setup, the needle electrodes (called also "subdermal needles") were out of sight right from the beginning.

The layout (the used setup) of the recording system can be seen in Figure 3 and in Figure 4 can be seen the recording process.

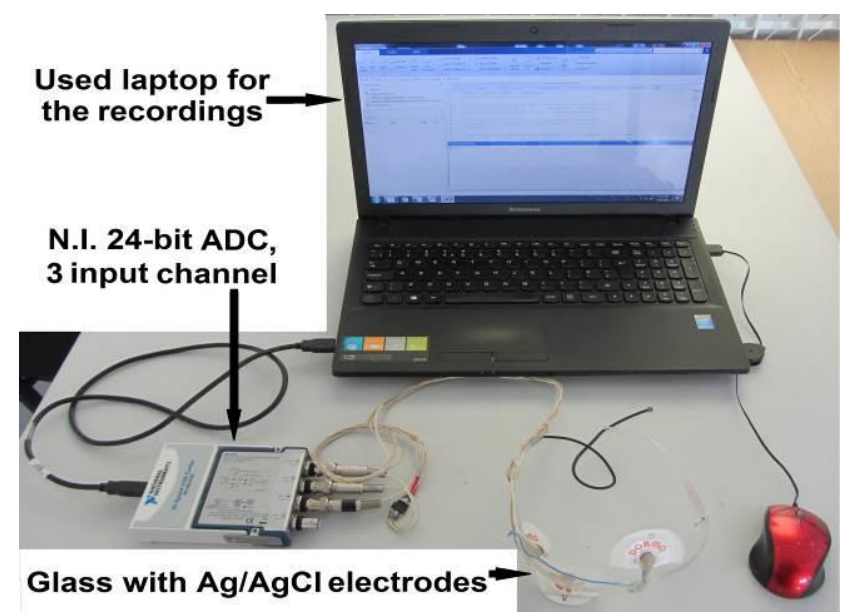

Fig. 3. The used setup for recording the EOG bio-signals

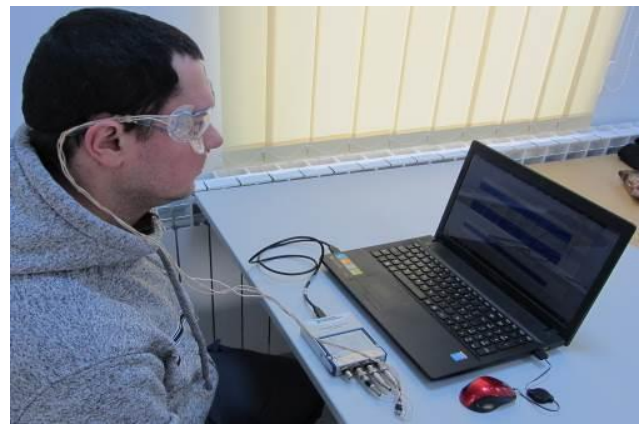

Fig. 4. Recording the EOG signals

\section{SIGNAL PROCESSING AND CLASSIFICATION}

Signals acquired with the system described above had been recorded for 50 independent measurements for each eye movement (right, left, down and up) from all three sensors. An example of the recorded signal is illustrated in Figure 5. As it can be seen from Figure 5, the signals are considerably noisy and had to be filtered which we achieved with median filters. After filtering the signals we obtained the shapes presented in Figures 6-7.

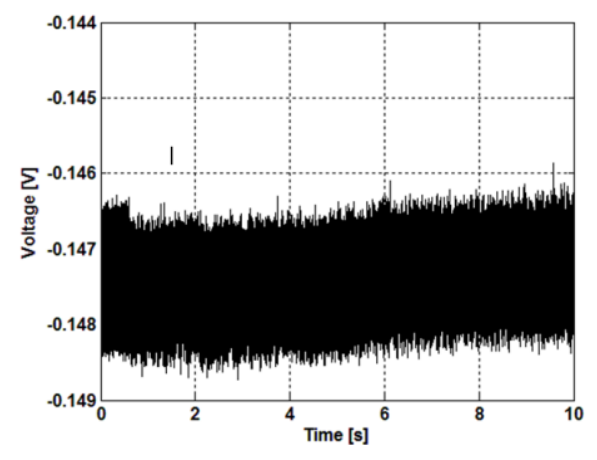

Fig. 5. Example of unprocessed recorded signal 

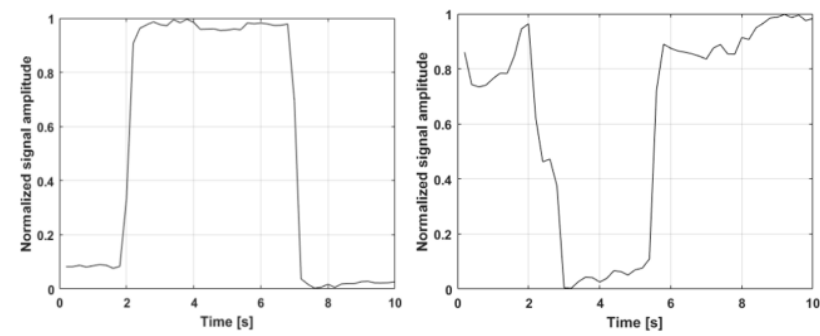

Fig. 6. Type T1 signal (left) and type T2 (right)
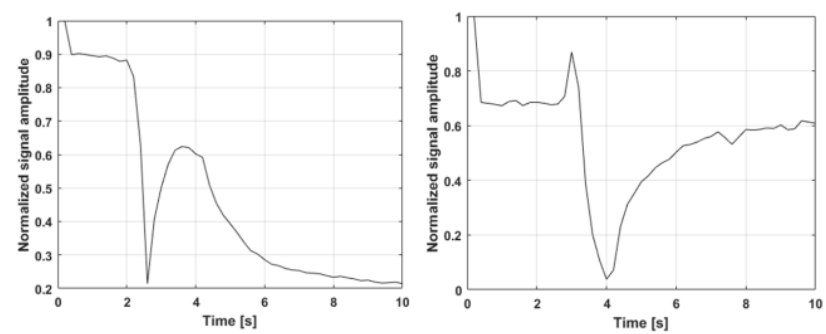

Fig. 7. Type T3 signal (left) and type T4 (right)

In order to recognize and classify the signals and identify the type of movement of the eye from the signals of the three sensors we analyzed the signal shapes and divided them into 4 categories. So for example if sensor 0 will give a signal of type T1 and sensors 1 and 2 will give signals of type T2, we can assume that the eye movement was in the direction " $R$ " (right). The signals were further down sampled in order to reduce processing time and memory space.

For recognition and classification purposes we decided to use an artificial neural network and analyze the obtained results. Artificial neural networks had been used for pattern recognition for more than 4 decades with more or less success. In the last decade a new approach called Deep Learning has been introduced after the works presented in [27] and [28] among others. Deep Learning algorithms have a range of methods like Deep Belief Networks, Restricted Boltzmann Machines and Deep Autoencoders to mention just some of them. These methods proved to be very useful in a large number of applications, a lot of worldwide companies employing them in their software products. In the last releases of Matlab Neural Network Toolbox a Deep Learning module based on aotuencoders had been also included. We used this module for our analysis [29].

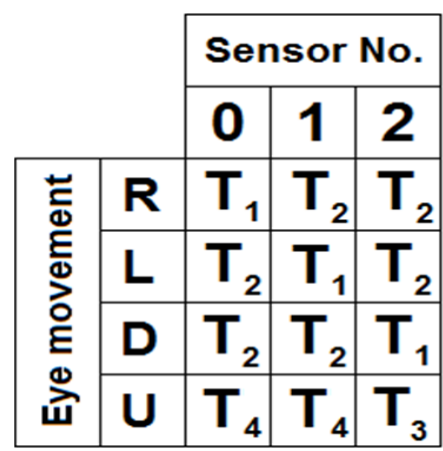

Fig. 8. Sensor signals corresponding to eye movements.
T1, T2, T3, T4 types of signal shapes,

Eye movement $\mathrm{R}$ - right, L - left, D - down, U - up.

Autoencoders perform unsupervised learning to extract features of the input data. In our network we trained two autoencoders and a softmax layer with 540 input samples consisting of 50 samples each and representing all four types of signals (T1, T2, T3 T4). We left aside 60 samples which we used to test the network after it had been trained. After the unsupervised learning session the 2 autoencoders and the softmax layer were stacked together to form the final network. For the first autoencoder we used 30 neurons for the second 10 neurons and for the softmax layer we used 4 neurons to match the outputs. The output consist of a column vector for 4 elements in double precision format with values between 0 and 1 , representing the four types of signal shapes classified as above in Figure 8. The index of the maximal valued element of the output vector shows in which type the input signal can be classified. The developed neural network is shown in Figure 9.

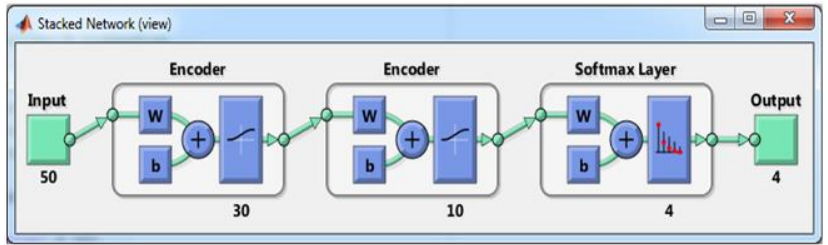

Fig. 9. Neural network used for signal classification

We trained the network several times always obtaining a performance of $100 \%$. This is shown in the confusion matrix in Figure 10. The numbers in the green squares show the well classified samples and those in red squares show the wrongly classified samples.

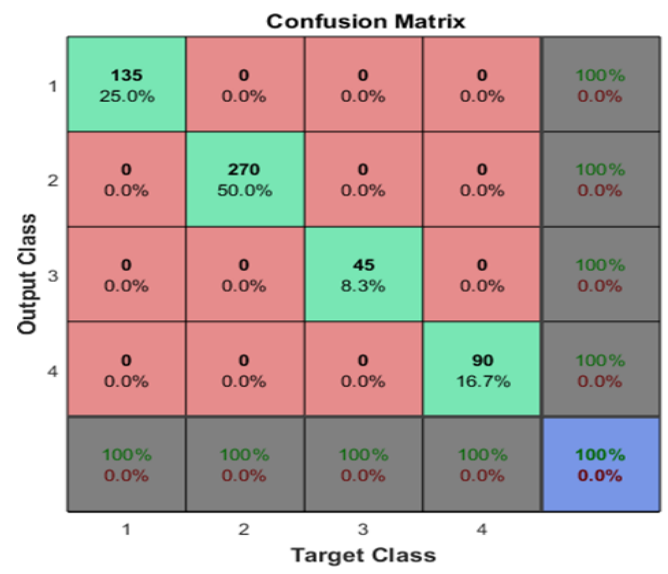

Fig. 10. Sensor signals corresponding to eye movements

However the results for the test samples show (in the best cases) that there had been six samples which were wrongly classified, as it can be seen in figure 11. These give an overall performance of just $90 \%$. 


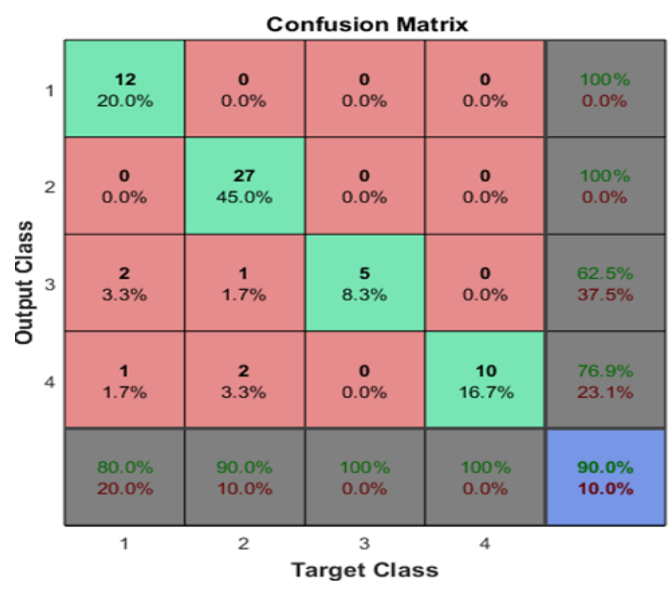

Fig. 11. Confusion matrix for test session

Analyzing the samples one by one we could find which were the wrongly classified samples. These samples are shown in Figures 12 to 17.

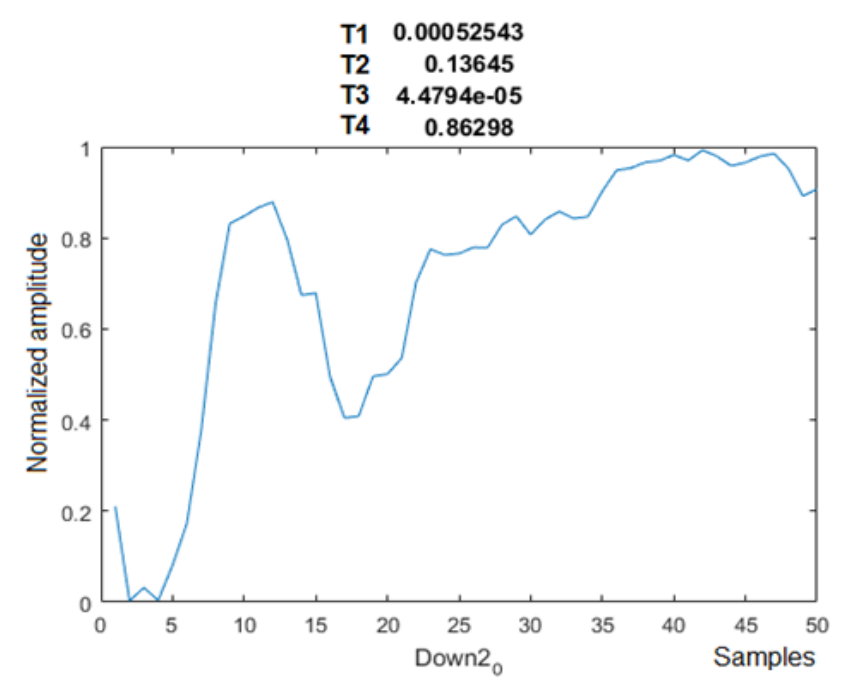

Fig. 12. Test sample 2, sensor 0

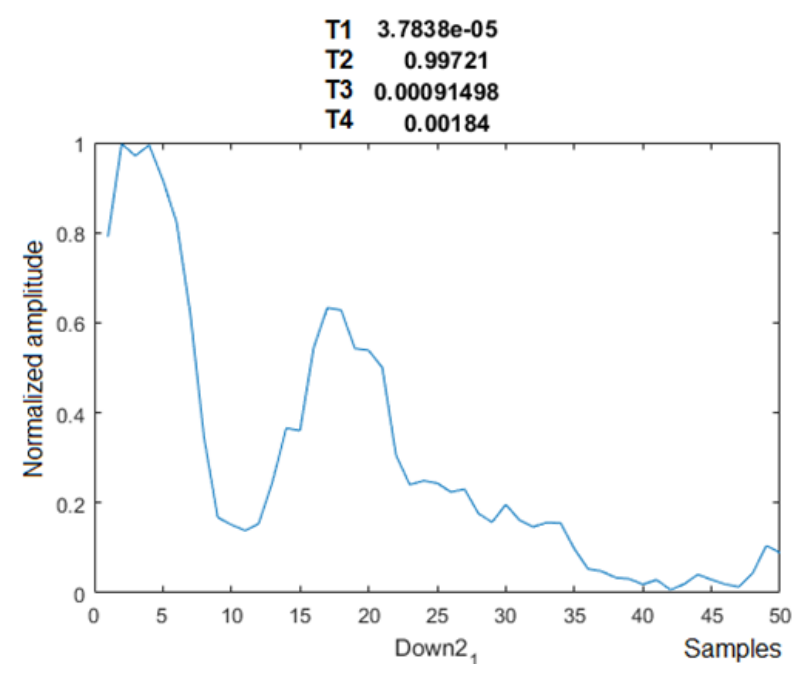

Fig. 13. Test sample 2, sensor 1

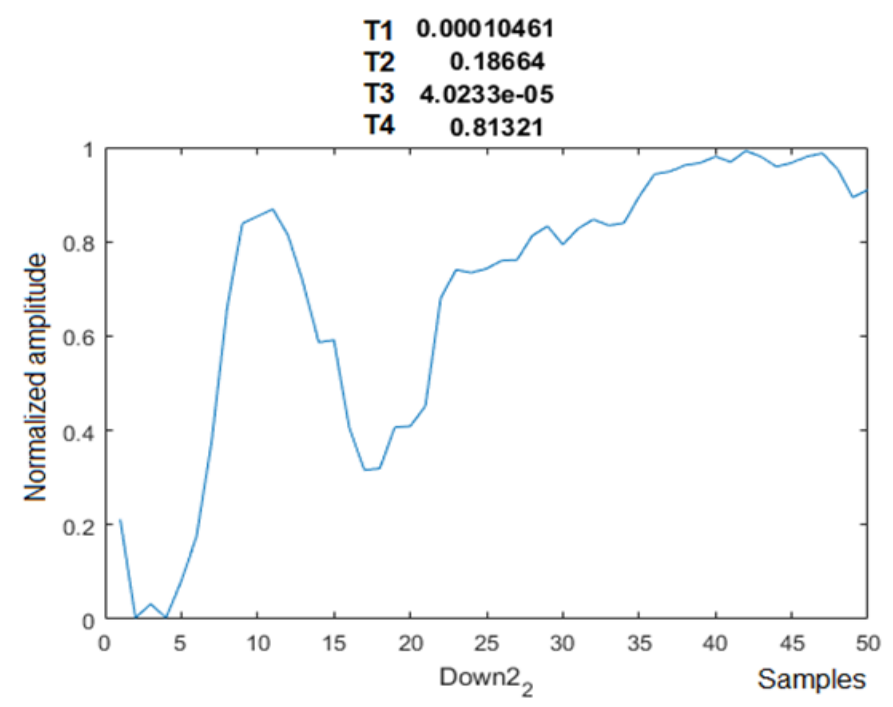

Fig. 14. Test sample 2, sensor 2

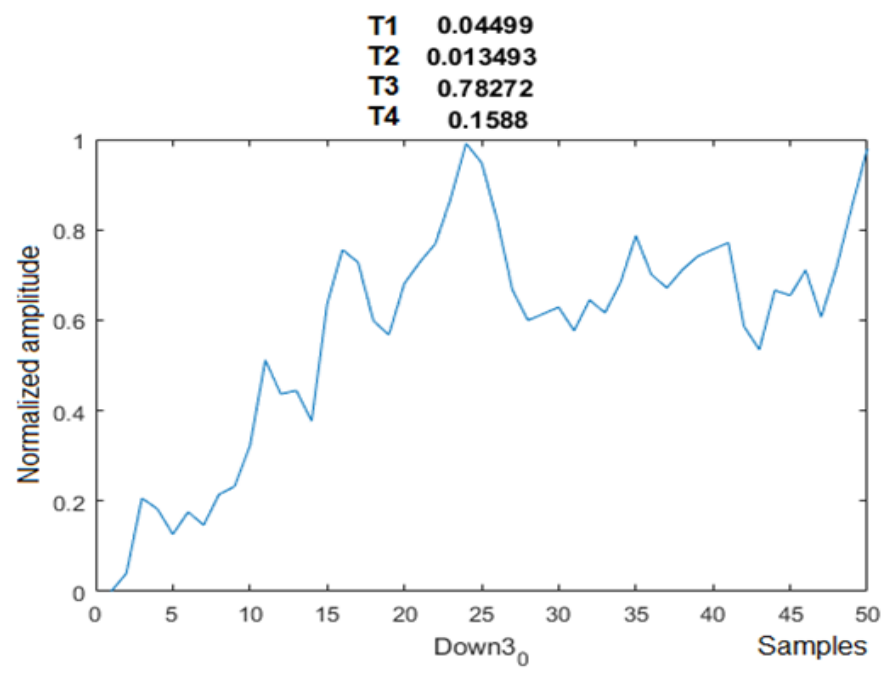

Fig. 15. Test sample 3, sensor 0

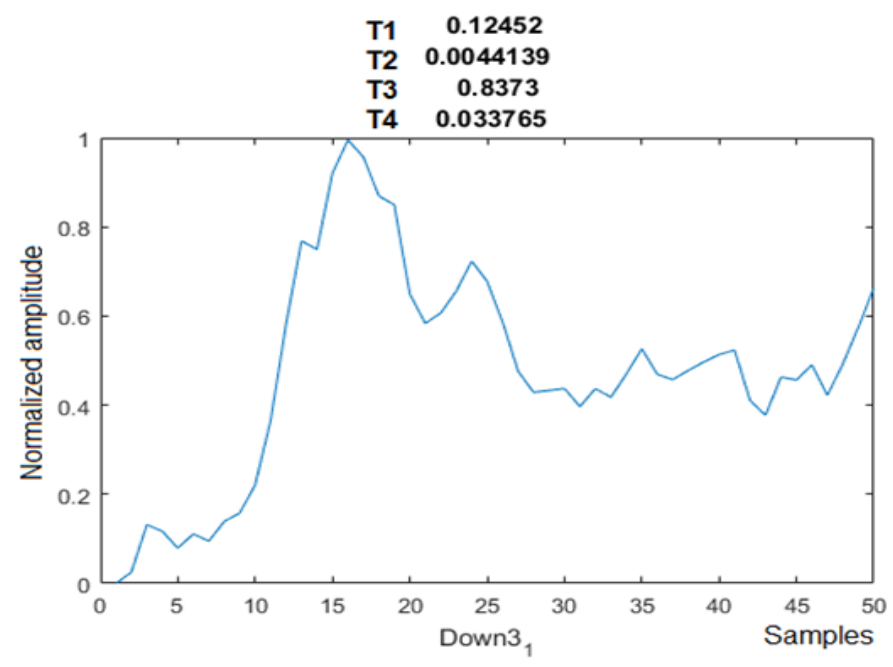

Fig. 16. Test sample 3, sensor 1 


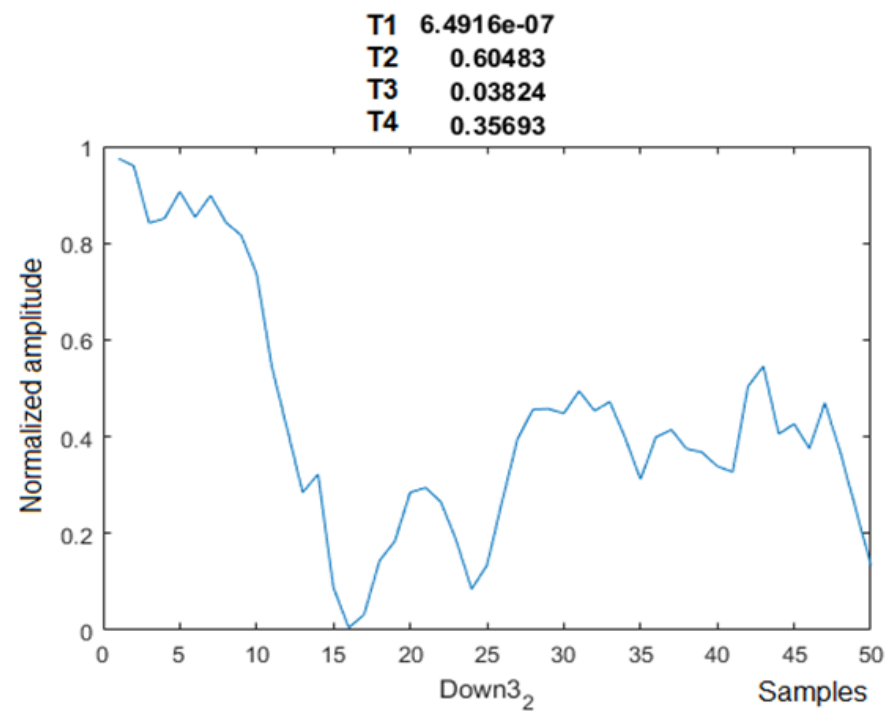

Fig. 17. Test sample 3, sensor 2

On the diagrams in figure 12 to 17 we also represented above the diagram, the output vector values which show in which type the wrongly classified signals have been included (see maximal value).

\section{CONCLUSIONS}

As we can see from these diagrams the signal shapes are very different from the common type of shape which indicates that these measurements were affected with a much larger noise than the other samples. This can have as a cause the fact that the electrode was not well attached or some contact failure could have occurred. We can also observe that all the wrongly classified signals are for the case of "down" eye movement. This can show that the positions of the electrodes are not entirely fit for sensing the activation of the muscles for the down eye movement. These errors can be corrected in future experiments finding better places for the electrodes on the glasses.

The overall conclusion is that the experimental system worked satisfactory well with the observation that care must be taken to firmly put the electrodes in the proper places. The classification using the neural network worked very well and classified well the vast majority of the signals. We can argue that those signals which were wrongly classified by the neural network could not be classified properly even by a human analyst.

\section{ACKNOWLEDGMENT}

Software and hardware elements used in this research had been provided through the "Micromodel HURO/0802/100" project of the European Union.

\section{REFERENCES}

[1] A. Pal, A. K. Gautam, Y. N. Singh, "Evaluation of Bioelectric Signals for Human Recognition," in Procedia Computer Science 48, 2015, pp. 746-752.
[2] R. Ying, C. E. Wall, "A method for discrimination of noise and EMG signal regions recorded during rhythmic behaviors," in Journal of Biomechanics 49, 2016, pp. 4113-4118.

[3] J. Franzen, K. Brinkmann, "Wanting and liking in dysphoria: cardiovascular and facial EMG responses during incentive processing," in Biological Psychology 121, 2016, pp. 19-29.

[4] O. Barzilay, A. Wolf, "A fast implementation for EMG signal linear envelope computation," in Journal of Electromyography and Kinesiology 21, 2011, pp. 678-682.

[5] V. K. Mishra, V. Bajaj, A. Kumar, D. Sharma, G.K. Singh, "An efficient method for analysis of EMG signals using improved empirical mode decomposition," in Int. J. Electron. Commun. (AEÜ) 72, 2017, pp. 200209.

[6] Í. Corera, A. Malanda, J. Rodriguez-Falces, S. Porta, J. Navallas, "Motor unit profile: A new way to describe the scanning-EMG potential," in Biomedical Signal Processing and Control 34, 2017, pp. 64-73.

[7] M. Marouf, L. Saranovac, G. Vukomanovic, "Algorithm for EMG noise level approximation in ECG signals," in Biomedical Signal Processing and Control 34, 2017, pp. 158-165.

[8] D. Yang, H. Zhang, Y. Gu, H. Liu, "Accurate EMG onset detection in pathological, weak and noisy myoelectric signals," in Biomedical Signal Processing and Control 33, 2017, pp. 306-315.

[9] J. Hu, C. Wang, M. Wu, Y. Du, Y. He, J. She, "Removal of EOG and EMG artifacts from EEG using combination of functional link neural network and adaptive neural fuzzy inference system," in Neurocomputing 151, 2015, pp. 278-287.

[10] H.-A. T. Nguyen, J. Musson, F. Li, W. Wang, G. Zhang, R. Xu et al., "EOG artifact removal using a wavelet neural network," in Neurocomputing 97, 2012, pp. 374-389.

[11] C. Burger, D. J. van den Heever, "Removal of EOG artefacts by combining wavelet neural network andindependent component analysis," in Biomedical Signal Processing and Control 15, 2015, pp. 67-79.

[12] M. Fatourechi, A. Bashashati, R. K. Ward, G. E. Birch, "EMG and EOG artifacts in brain computer interface systems: A survey," in Clinical Neurophysiology 118, 2007, pp. 480-494.

[13] M. A. Klados, P. D. Bamidis, "A semi-simulated EEG/EOG dataset for the comparison of EOG artifact rejection techniques," in Data in Brief 8, 2016, pp. 1004-1006.

[14] J. A. E. Christensen, L. Kempfner, H. L. Leonthin, M. Hvidtfelt, M. Nikolic, B. R. Kornum, P. Jennum, "Novel method for evaluation of eye movements in patients with narcolepsy," Sleep Medicine xxx (2016) 110 , in press.

[15] A. Picot, S. Charbonnier, A. Caplier, "EOG-based drowsiness detection: Comparison between a fuzzy system and two supervised learning classifiers," in Proceedings of the 18th World Congress - The International Federation of Automatic Control, 2011.

[16] C. Rasch, J. J. Louviere, T. Teichert, "Using facial EMG and eye tracking to study integral affect in discrete choice experiments," in The Journal of Choice Modelling 14, 2015, pp. 32-47.

[17] Z. Lv, X. Wu, M. Li, D. Zhang, "A novel eye movement detection algorithm for EOG driven human computer interface," in Pattern Recognition Letters 31, 2010, pp. 1041-1047.

[18] A. L. C. Bissoli, M. M. Sime, T. F. Bastos-Filho, "Using sEMG, EOG and VOG to control an intelligent environment," in IFACPapersOnLine, 2016, pp. 210-215.

[19] L. Y. Deng, C.-L. Hsu, T.-C. Lin, J.-S. Tuan, S.-M. Chang, "EOG-based human-computer interface system development," in Expert Systems with Applications 37, 2010, pp. 3337-3343.

[20] R. Barea, L. Boquete, S. Ortega, E. López, J.M. Rodríguez-Ascariz, "EOG-based eye movements codification for human computer interaction," in Expert Systems with Applications 39, 2012, pp. 26772683.

[21] C.-C. Postelnicu, F. Girbacia, D. Talaba, "EOG-based visual navigation interface development," in Expert Systems with Applications 39, 2012, pp. 10857-10866. 
[22] X. Guo, W. Pei, Y. Wang, Y. Chen, H. Zhang, X. Wu et al., "A humanmachine interface based on single channel EOG and patchable sensor," in Biomedical Signal Processing and Control 30, 2016, pp. 98-105.

[23] S. Aungsakul, A. Phinyomark, P. Phukpattaranont, C. Limsakul, "Evaluating feature extraction methods of electrooculography (EOG) signal for human-computer interface," in Procedia Engineering 32, 2012, pp. 246-252.

[24] S. Soltani, A. Mahnamn, "A practical efficient human computer interface based on saccadic eye movements for people with disabilities," in Computers in Biology and Medicine 70, 2016, pp. 163-173.

[25] http://www.wisegeek.com/what-are-the-different-types-of-eegelectrodes.htm, accessed on 22 February 2017.
[26] R. B. Nagy, T. Vesselenyi, F. Popentiu-Vladicescu, "Research on recording and filtering electromyogram (EMG) signals," in Nonconventional Technologies Review, 2015, pp. 21-25.

[27] G. E. Hinton, S. Osindero, and Y.-W. Teh. "A fast learning algorithm for deep belief nets," in Neural computation 18, 2006, pp. 1527-1554.

[28] Y. Bengio, P. Lamblin, D. Popovici, and H. Larochelle. "Greedy layerwise training of deep networks," in Advances in Neural Information Processing Systems, 2007.

[29] ***. MATLAB. - Mathworks, Users Manual, Neural Network Toolbox, Deep Learning, Autoencoders, 2016. 